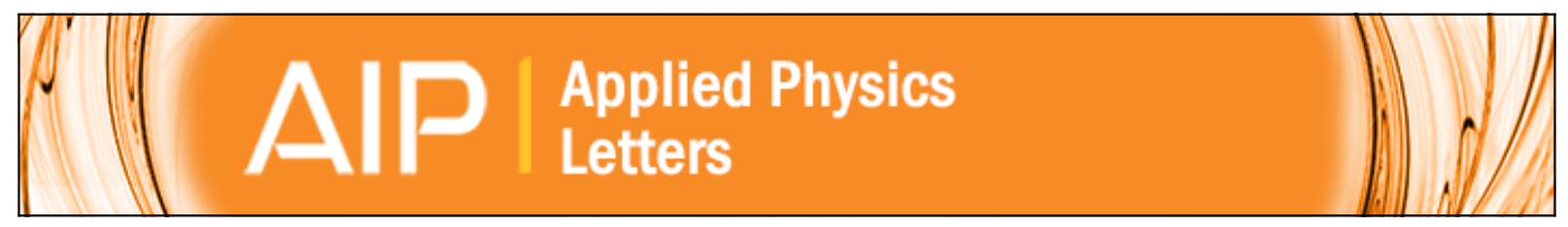

\title{
Emission wavelength of AIGaAsGaAs multiple quantum well lasers
}

P. Blood, E. D. Fletcher, P. J. Hulyer, and P. M. Smowton

Citation: Applied Physics Letters 48, 1111 (1986); doi: 10.1063/1.96613

View online: http://dx.doi.org/10.1063/1.96613

View Table of Contents: http://scitation.aip.org/content/aip/journal/apl/48/17?ver=pdfcov

Published by the AIP Publishing

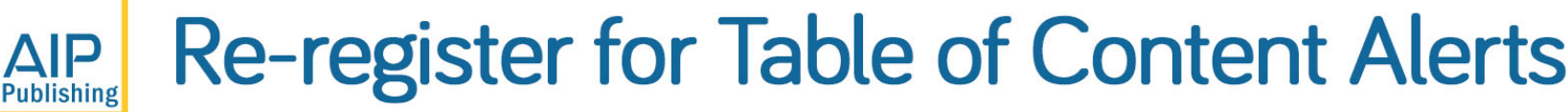

\section{Create a profile.}




\title{
Emission wavelength of AIGaAs-GaAs multiple quantum well lasers
}

\author{
P. Blood, E. D. Fletcher, P. J. Hulyer, and P. M. Smowton ${ }^{\text {a) }}$ \\ Philips Research Laboratories, Redhill, Surrey RH1 5HA, England
}

(Received 6 February 1986; accepted for publication 4 March 1986)

\begin{abstract}
We have recorded spontaneous emission spectra from multiple quantum well lasers grown by molecular beam epitaxy with 25 - $\AA$-wide GaAs wells by opening a window in the top contact stripe. These spectra have a low-energy tail and consequently the gain spectra derived from them show that laser emission occurs at a lower photon energy than the lowest energy confined particle transition. The observed laser wavelength and threshold current are consistent with the position of the peak in the gain spectrum.
\end{abstract}

It is commonly observed that laser emission from AlGaAs/GaAs quantum well structures occurs at longer wavelengths than that associated with the lowest energy confined particle transition. In early experiments, using optically pumped structures grown by metalorganic chemical vapor deposition (MOCVD), this phenomenon was ascribed to participation of longitudinal optic (LO) phonons ${ }^{1}$ in the emission process. Subsequently in electrical injection single quantum well (SQW) lasers, also grown by MOCVD by Hersee et al., it was noted that the laser wavelengths corresponded to transition energies of wells $30 \AA$ wider than predicted or measured ${ }^{3}$ and this apparent increase in well width was attributed to composition fluctuations in the barrier as proposed by Coleman et $a l .{ }^{4}$ Coleman et al. noted that the peak positions in the absorption spectrum of a multiple quantum well (MQW) structure with AlGaAs barriers corresponded to a well width of $\sim 93 \AA$ rather than the width of $80 \AA$ predicted from the growth and ascribed this difference to clustering in the alloy barriers. With a series of MQW injection lasers grown by molecular beam epitaxy (MBE) we observed that laser emission occurred at wavelengths 15 to $23 \mathrm{~nm}$ longer than that predicted for the confined particle transitions from knowledge of the well width $\left(L_{z}\right)$ derived from the growth rate calibrated by electron diffraction oscillations. ${ }^{5}$ We were also able to record photovoltage absorption spectra from the active region of some of these device layers and when corrected for the exciton binding energy the peak positions agreed with the predicted transition wavelengths within experimental error. ${ }^{5}$ This agreement contrasts with the observations in Ref. 4 and suggests that clustering in the barriers as proposed by Coleman et al. does not occur in our samples. Furthermore, photovoltage spectra on the material used for our MQW devices described in Ref. 6 also give peak positions at shorter wavelengths than the observed laser transitions for all the structures, and we conclude that there is a real difference between the wavelengths of the absorption peaks and laser transitions. Since these measurements are made on the same structures this comparison is independent of any theoretical model, composition grading at the interfaces, and errors in the values of well width and barrier composition. Although such a wavelength

\footnotetext{
" Industrial Trainee from Dept. of Physics, Electronics and Electrical Engineering, UWIST, P. O. Box 25, Cardiff.
}

difference is observed in conventional lasers due to the impurity "tail" states below the band edge, ${ }^{7}$ it is not expected in an ideal quantum well structure because of the rectangular density of states function. Theoretical gain spectra for such uncoupled quantum wells have maxima at the same photon energy as the lowest confined particle transition and a sharp low-energy edge on the spontaneous spectrum. ${ }^{8}$

To investigate further the reason for this wavelength difference we have recorded spectra of the spontaneous emission through a window in the top contact of stripe geometry MQW lasers and have used these to calculate gain spectra as a function of quasi-Fermi level separation. The results presented in this paper show that due to a low-energy tail on the spontaneous spectrum the maximum gain occurs at a lower photon energy than the confined particle transition and that the observed laser emission wavelengths are consistent with these data.

The devices had 25 - $\AA$-wide GaAs wells separated by 75 $\AA$-wide $\mathrm{Al}_{0.35} \mathrm{Ga}_{0.65} \mathrm{As}$ barriers, with the MQW system being located at the center of a 4000 - $\AA$-wide graded waveguide with $\mathrm{Al}_{0.6} \mathrm{Ga}_{0.4}$ As outer cladding regions. The active region was not intentionally doped (the background level was $p \approx 3 \times 10^{16} \mathrm{~cm}^{-3}$ measured by capacitance-voltage profiling ). Full details of the MBE growth and characterization of the structures are given in Ref. 6. A window about $10 \mu \mathrm{m}$ wide was opened along the full length of the AuZn $50-\mu \mathrm{m}$ wide $p$-type contact of oxide stripe laser devices, and the top GaAs layer removed in the window so that the emission from the active region could be observed without any intervening absorber. The devices were mounted layer side up and operated pulsed at room temperature with a duty cycle of $\approx 2 \times 10^{-4}$ and pulse width $\approx 200 \mathrm{~ns}$. Radiation from the window was focused onto the vertical slits of a $1-\mathrm{m}$ grating spectrometer with care taken to eliminate radiation from the ends of the device by using a long device and narrowing the horizontal slits appropriately.

Figure 1 shows the spontaneous emission energy spectrum from a 20 well laser $780 \mu \mathrm{m}$ long operated at 0.85 of the threshold current $\left(I_{\mathrm{th}}\right)$. The positions of the $n=1$ excitonic absorption peaks obtained from photovoltage absorption spectra ${ }^{9}$ are indicated, and adding the exciton binding energy $^{10}$ gives the $n=1(e-\mathrm{hh})$ subband transition energy at $1.685 \mathrm{eV}$. At high currents the individual excitonic peaks are not apparent on the emission spectrum, probably because of 


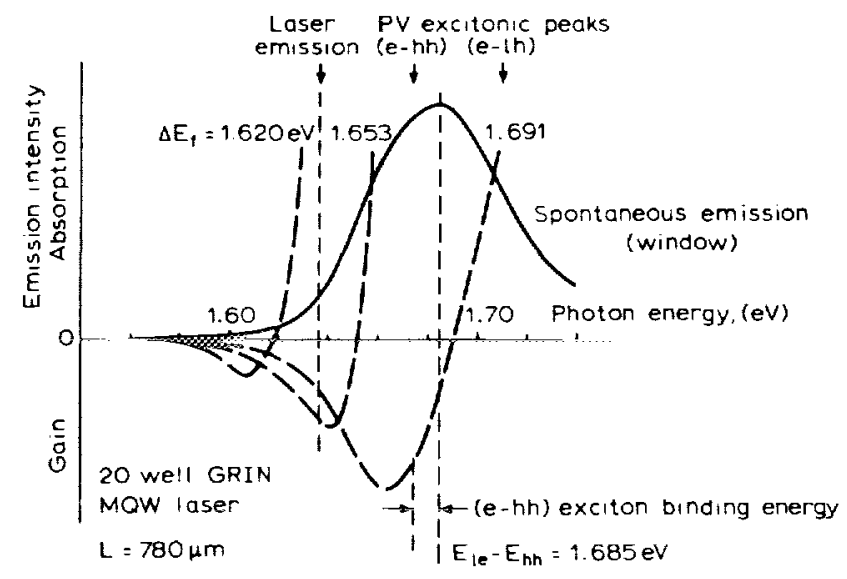

FIG. 1. Spontaneous emission spectrum $r_{\text {spon }}(E)$ observed through the window in the contact stripe of an oxide stripe MQW laser with $20 \mathrm{GaAs}$ wells $25 A$ wide; the device is $780 \mu \mathrm{m}$ long. The energies of the $n=1(e-h \mathrm{~h})$ and ( $e$-lh) excitonic absorption peaks observed by photovoltage spectroscopy (PV) on the active region of the device layer, and the observed laser emission energy are indicated at the top of the figure. The energy separation of the $n=1 e$ and hh confined particle states has been obtained by adding the exciton binding energy $(\approx 9 \mathrm{meV})$ to the PV peak energy. Absorption spectra calculated from $r_{\text {spon }}(E)$ for three values of $\Delta E_{f}$ using Eq. (1) are shown. The negative going peaks represent the gain spectrum for each $\Delta E_{f}$

screening at high injected carrier densities, and it can be seen that the emission peak corresponds to the $e-h$ h transition. This transition provides greater gain than the $e-$ Ih transition and is responsible for the operation of the laser. From a current of $0.01 I_{\text {th }}$ to $\approx I_{\text {th }}$ the spontaneous emission peak moves by only $6 \mathrm{meV}$ ( $25 \AA$ ) to shorter wavelengths, probably due to band filling. As indicated on Fig. 1, laser emission for this device occurs at an energy $42 \mathrm{meV}$ lower than the spontaneous emission peak $(\approx 200 \AA$ longer wavelength).

The absorption spectrum $\alpha(E)$ in arbitrary units was calculated from the spontaneous emission spectrum $r_{\text {spon }}(E)$ (in relative units) using the relation "

$$
\alpha(E)=-\frac{C}{E^{2}}\left[1-\exp \left(\frac{E-\Delta E_{f}}{k T}\right)\right] r_{\text {spon }}(E),
$$

where $\Delta E_{f}$ is the quasi-Fermi level separation and the constant $C$ was set equal to unity. Figure 1 shows absorption spectra calculated for three values of $\Delta E_{f}$. These spectra have gain peaks at energies below the observed energy of the lowest confined particle transitions due to the tail on the emission spectrum, and in Fig. 2 we have plotted the wavelength of the gain peak as a function of quasi-Fermi level separation. Although there are small changes in the shape of $r_{\text {spon }}(E)$ with drive current we find that these changes have a negligible effect on the position of the gain peak, so it is satisfactory to use the same emission spectrum in calculating all points in Fig. 2. For this device laser emission occurs at $7570 \AA$ which implies that $\Delta E_{f}=1.648 \mathrm{eV}$.

We now wish to estimate whether this Fermi level separation is consistent with the threshold current density $J_{\text {th }} / d=9 \times 10^{4} \mathrm{~A} \mathrm{~cm}^{-2} \mu \mathrm{m}^{-1}$ measured for this device, with the width $d$ being the total well width $20 \times 25 \AA$. Assuming an ideal rectangular density of states function

$$
\rho_{c}=m_{c} / \pi h^{2} L_{z} \quad E \geqslant E_{\mathrm{le}}
$$

corresponding to the well width $L_{z}$, using $m_{c}=0.065 m_{0}$

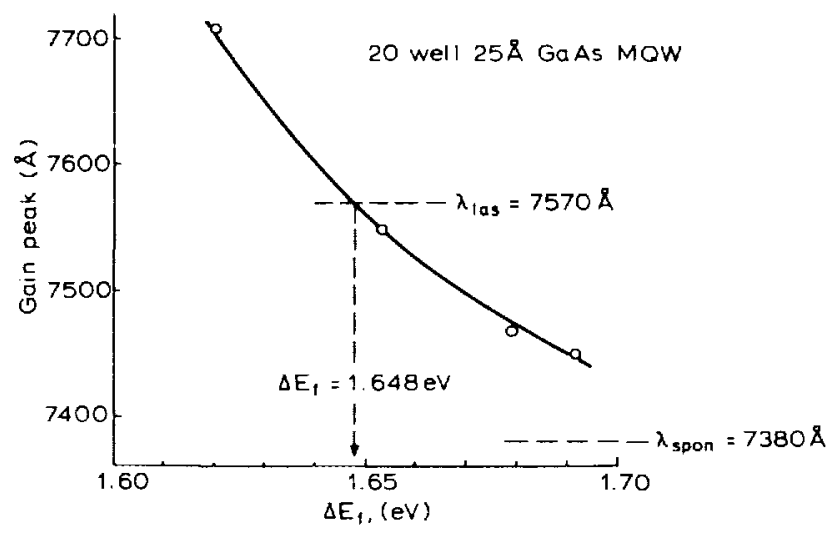

FIG. 2. Plot of the wavelength of the gain peaks, obtained from Fig. 1 and similar data, as a function of quasi-Fermi level separation. The observed wavelengths of laser emission ( $\left.\lambda_{\text {las }}\right)$ and the spontaneous emission peak $\left(\lambda_{\text {spon }}\right)$ are indicated; $\lambda_{\text {las }}$ tends to $\lambda_{\text {spon }}$ at high injection.

and a small value of heavy hole effective mass in the plane of the wells $m_{v}=0.15 m_{0}$ since the well is narrow, ${ }^{12}$ we calculate $n=p=1.7 \times 10^{18} \mathrm{~cm}^{-3}$ for $\Delta E_{f}=1.648 \mathrm{eV}$ at threshold. The radiative recombination constant $B$ is not known for these wells, though Fig. 2 of Ref. 13 suggests $B \approx 3.3 \times 10^{-10} \mathrm{~cm}^{3} \mathrm{~s}^{-1}$ for wells about $25 \AA$ wide (using $\tau^{-1}=B n$ ). In bulk GaAs $B$ decreases with increasing injected minority-carrier density ${ }^{7}$ so we have taken $B=2.0 \times 10^{-10} \mathrm{~cm}^{3} \mathrm{~s}^{-1}$ giving a spontaneous current density $J_{\text {spon }}=B e n p \approx 1.0 \times 10^{4} \mathrm{~A} \mathrm{~cm}^{-2} \mu \mathrm{m}^{-1}$. This is about $25 \%$ of the measured threshold current (including a correction for current spreading) and could imply a very low internal efficiency $\left(\eta_{i}\right)$ which is unlikely, or that the assumed value of $B$ is too low, or that the density of states is incorrect due to inadequate knowledge of the effective mass or due to tail states.

The observation of laser emission at photon energies below the $n=1$ ( $e-h$ h) transition energy and the extended low-energy tail on the spontaneous spectrum (Fig. 1) suggest that states exist at electron and hole energies below the $n=1$ confined electron and hole states of the ideal quantum well. The resulting increase in density of states at low energy means the injected carrier density must be higher than calculated above to achieve the same quasi-Fermi level separation. Using a modified density of states function with a triangular tail which accounts approximately for the low-energy spontaneous emission tail we then calculate $n=p=2.50 \times 10^{18} \mathrm{~cm}^{-3}$ which, with $B=2 \times 10^{-10}$ $\mathrm{cm}^{3} \mathrm{~s}^{-1}$, gives $J_{\text {spon }} \approx 2.0 \times 10^{4} \mathrm{~A} \mathrm{~cm}^{-2} \mu \mathrm{m}^{-1}$, a value which is $0.5 J_{\mathrm{th}} / d$, after allowing for current spreading, close to our estimates of $\eta_{i}$ from devices of various lengths. We conclude that within the uncertainties in our knowledge of $B$ and the true density of states function, the quasi-Fermi level separation of $1.648 \mathrm{eV}$ is not inconsistent with the measured threshold current of the device.

The observed laser emission wavelength with respect to the spontaneous emission peak is determined by the threshold current density of the particular device, and by the shape of the spontaneous spectrum at low energies. Figure 2 shows that the wavelength difference increases as $\Delta E_{f}$ decreases and so devices with low threshold current density per unit 
volume operating with a low gain have the largest shift of laser emission to longer wavelength. We observe a low-energy tail on the spontaneous spectra of all the quantum well laser structures we have examined: six so far. In particular, the devices used in Ref. 6 show that $J_{\text {th }} / d$ decreases as the number of wells $(N)$ increases for a fixed well width and we also observe an increasing shift of laser emission to long wavelengths as $N$ increases which is accounted for by the measured values of $J_{\mathrm{th}} / d$ and gain spectra calculated from $r_{\text {spon }}(E)$ using Eq. (1).

The spontaneous emission spectra observed from our devices show that there are states accessible at photon energies below the ideal $n=1(e-h h)$ confined particle transition energy. The origin of these states is not clear at the present time, though many body effects at high injection have been shown to give a tail on the spectrum ${ }^{14}$ and intraband relaxation broadens the gain spectrum. ${ }^{15}$ Fluctuations in the well width may have a similar effect. Published results for gain spectra measured on quantum well lasers ${ }^{16,17}$ have a rounded, approximately symmetrical shape which is consistent with our observed emission spectra and the shape of the derived gain spectra in Fig. 1, and contrast with the form of gain spectra which have been calculated from ideal density of states functions using $k$ selection. ${ }^{8,18}$

While it is possible for the laser emission wavelength to differ from its anticipated value by an error in the value of $L_{z}$ or by grading or clustering in the barriers, our results show that this is not necessarily the direct cause of the shift in laser wavelength. By measuring absorption spectra, spontaneous emission spectra through a contact window, and laser emission wavelength on a given device structure we have shown that in our MBE material the absorption peak positions agree with the well width derived from the growth conditions as described in Ref. 5, and that the laser emission occurs at a longer wavelength than the absorption peak due to the recombination we observe on the spontaneous spectrum at low photon energies. If this low-energy emission is due to an intrinsic process such as many body interactions, then this wavelength lengthening mechanism will occur in all quantum well lasers.

We thank $\mathrm{K}$. Woodbridge for growing the structures used in this work and P. Dawson, G. Duggan, and H. I. Ralph for illuminating discussions.

'J. J. Coleman, P. D. Dapkus, D. R. Clarke, M. D. Camras, and N. Holonyak, Jr., Appl. Phys. Lett. 39, 864 (1981).

${ }^{2}$ S. Hersee, B. de Cremoux, and J. P. Duchemin, Appl. Phys. Lett. 44, 476 (1984).

${ }^{3}$ S. D. Hersee, M. Krakowski, R. Blondeau, M. Baldy, B. de Cremoux, and J. P. Duchemin, J. Cryst. Growth 68, 383 (1984).

${ }^{4} J$. J. Coleman, P. D. Dapkus, M. D. Camras, N. Holonyak, Jr., W. D. Laidig, T. S. Low, M. S. Burroughs, and K. Hess, J. Appl. Phys. 52, 7291 (1981).

${ }^{5}$ K. Woodbridge, P. Blood, E. D. Fletcher, and P. J. Hulyer, Appl. Phys. Lett. 45, 16 (1984).

${ }^{6}$ P. Blood, E. D. Fletcher, and K. Woodbridge, Appl. Phys. Lett. 47, 193 (1985).

${ }^{7}$ F. Stern, J. Appl. Phys. 47, 5382 (1976).

${ }^{8}$ See, for example, T. Ohtoshi, K. Uomi, N. Chinone, T. Kajimura, and Y. Murayama, J. Appl. Phys. 57, 992 (1985).

${ }^{9}$ P. Blood, E. D. Fletcher, K. Woodbridge, P. Dawson, and P. J. Hulyer, in International Symposium on GaAsand Related Compounds, Biarritz 1984, Institute of Physics Conf. Ser. 74, edited by B. de Cremoux (Institute of Physics, Bristol, 1985), p. 427, and Ref. 5.

${ }^{10} \mathrm{R}$. L. Greene and K. K. Bajaj, Solid State Commun. 45, 831 (1983),

"G. Lasher and F. Stern, Phys. Rev. A 133, 553 (1964) [Eqs. (8) and (9)].

${ }^{12}$ A. Fasolino and M. Altarelli, in Tuo Dimensional Systems, Heterostructures and Superlattices, edited by G. Bauer, F. Kuchar, and H. Heinrich (Springer, Berlin, 1984), p. 176 [ Eq. (4) of this paper gives $m_{\mathrm{hh}}=0.12$ for an infinite barrier].

${ }^{13}$ Y. Arakawa, H. Sakaki, M. Nishioka, and J. Yoshino, Appl. Phys. Lett. 46, 519 (1985).

${ }^{14}$ S. Tarucha, H. Kobayashi, Y. Horikoshi, and H. Okamoto, Jpn. J. Appl. Phys. 23, 874 (1984).

${ }^{15} \mathrm{M}$. Yamada, S. Ogita, M. Yamagishi, and K. Tabata, IEEE J. Quantum Electron. QE-21, 640 (1985).

${ }^{16}$ N. K. Dutta, R. L. Hartman, and W. T. Tsang, IEEE J. Quantum Electron. QE-19, 1243 (1983).

${ }^{17}$ H. Kobayashi, H. Iwamura, T. Saku, and K. Otsuka, Electron. Lett. 19, 166 (1983)

${ }^{18}$ D. Kasemset, C. S. Hong, N. B. Patel, and P. D. Dapkus, IEEE J. Quantum Electron. QE-19, 1025 ( 1983 ). 